Research Paper

\title{
The Role of CXCL12 Axis in Lung Metastasis of Colorectal Cancer
}

\author{
Meng Wang, Xuyang Yang, Mingtian Wei, Ziqiang Wang ${ }^{凶}$ \\ Department of Gastroenterological Surgery, West China Hospital, Sichuan University, Chengdu, 610041 \\ $\triangle$ Corresponding author: Ziqiang Wang MD, Department of Gastroenterological Surgery, West China hospital of Sichuan University, No.37 Guo Xue Xiang, \\ Chengdu, Sichuan Province, China, 610041. (Phone: +86-028-85422480, Fax: +86-028-85422480, E-mail: hxwangm@126.com) \\ (C) Ivyspring International Publisher. This is an open access article distributed under the terms of the Creative Commons Attribution (CC BY-NC) license \\ (https://creativecommons.org/licenses/by-nc/4.0/). See http://ivyspring.com/terms for full terms and conditions.
}

Received: 2018.03.30; Accepted: 2018.08.15; Published: 2018.10.10

\begin{abstract}
This study aimed to explore the mechanism of lung metastases of colorectal cancer. The expression of CXCR4, CXCR7, and CXCL12 in colorectal cancer and lung metastases was analyzed by the immunohistochemical (IHC) method. Further, the study aimed at getting new insights into the role of CXCL12 in lung metastasis. A total of 41 patients who underwent surgical resections for both colorectal cancer and lung metastasis were retrospectively selected for this study. Also, 41 patients who underwent lung resection for benign lung disease were selected as the control group. In the in vivo experiment, the mice were injected with exosomes isolated from CT26. The mice with and without injection were sacrificed 3 weeks later. The lung tissue was analyzed by the IHC method and quantitative real-time PCR. The expression of CXCR7 and CXCL1 2 was significantly higher in lung metastases than in paired primary lesions. The expression of CXCL12 was significantly higher in the noncancerous lung tissue of patients with CRC than in patients with benign lung disease. Also, the expression of CXCL12 in the lung was higher in mice with exosome education. The results inferred that the expression of CXCR7 and CXCL12 was significantly higher in lung metastases than in primary tumors. Their combination could promote colorectal cancer lung metastasis. The expression of CXCL12 was elevated before metastasis. And this effect was induced by exosomes.
\end{abstract}

Key words: colorectal cancer, CXCL12, exosome, lung metastasis

\section{Introduction}

In recent years, colorectal cancer (CRC) has become the fifth most common malignant tumors in China [1]. With the continuous development of medicine, the mortality rate of CRC has decreased by about $35 \%$ in the last 20 years [2]. However, tumor recurrence and metastasis are still common causes of treatment failure. Approximately $50 \%$ of patients with CRC after radical surgery of the primary lesion show tumor metastasis $[3,4]$. The most common metastatic sites of CRC are the liver and the lung. About $10 \%-25 \%$ of patients with CRC have lung metastasis [5]. Therefore, a better understanding of the mechanism of CRC metastasis may provide new means of cancer treatment.

Chemokine is a class of chemoattractive cytokines. After binding to corresponding receptors, they are critically involved in various physiological or pathological processes of angiogenesis, inflammation, tumor development, and so on [6]. CXCR4 and CXCR7 were both found to be expressed in CRC cells. Both were receptors of CXCL12, which was expressed in the most common sites of metastasis, such as lymph node, lung, and liver. In 2014, Yamada found that the expression of CXCR4 was higher in liver metastases than in primary tumors, and the prognosis was worse in patients with the expression of CXCR4 [7]. Miao found that CXCR7 promoted the growth of breast and lung cancers and tumor metastasis of lung [8]. However, which chemokine is important in the lung metastasis of CRC needs further exploration.

Exosomes with size ranging from 30 to $100 \mathrm{~nm}$ in diameter are shed by cells in the processes of cellular 
housekeeping and communication [9]. Exosomes can be secreted by both tumor and normal cells. In recent years, increasing evidence validated that exosomes could promote tumor development and metastasis [10]. Given the importance of CXCL12 in tumor metastasis, strong connections might exist between exosomes and CXCL12 expressed in the target organ.

This study analyzed the expression of CXCR4, CXCR7, and CXCL12 in CRC and lung metastases by the immunohistochemical (IHC) method to explore the mechanism of lung metastases of CRC. Further, the study aimed at getting new insights into the role of CXCL12 in lung metastasis.

\section{Materials and Methods}

\section{Patients}

A total of 41 patients who underwent surgical resections for both CRC and lung metastasis were retrospectively selected for this study. Their clinical and pathological characteristics are summarized in Table 1. Further, 41 patients who underwent lung resection for benign lung disease were selected as the control group; normal lung tissue samples were taken. All cases were confirmed by two senior pathologists. Formalin-fixed paraffin-embedded (FFPE) tissues from primary tumors and paired metastatic tumor specimens were taken from the Department of Pathology, West China Hospital, Sichuan University.

\section{Immunohistochemistry}

First, all slides were reviewed by two senior pathologists to confirm diagnosis, following which the expression of CXCR4, CXCR7, and CXCL12 was analyzed by the IHC method on FFPE tissue blocks. For CXCR4 (1:100 dilution, ab2074, rabbit anti-human, Abcam, Cambridge, UK), and CXCR7 (1:50 dilution, MAB42273 mouse monoclonal anti-Hu-CXCR7 [11G8], R\&D system), and CXCL12 (1:50 dilution, MAB 350 mouse monoclonal anti-Hu-CXCL12 [79018], R\&D system), heat-induced epitope retrieval was performed.

The image system comprised a camera (Olympus BX51, Japan). The expression of CXCR7, CXCR4, and CXCL12 was identified by the digital image analysis using the Image-Pro Plus 6.0 software (Image-Pro plus 6.0, Media Cybernetics Inc). The relative protein expression was quantified and defined as follows: density mean = density sum/area sum.

\section{Cell culture}

The BALB/c-derived CT26 mouse colon adenocarcinoma cell line was purchased from the cell bank of the Chinese Academy of Science (Shanghai, China). CT26 cells were cultured in RPMI 1640 at $37^{\circ} \mathrm{C}$ in a humidified atmosphere of $5 \% \mathrm{CO}_{2}$. The complete medium contained $10 \%$ fetal bovine serum, $100 \mathrm{U} / \mathrm{mL}$ penicillin, and $100 \mu \mathrm{g} / \mathrm{mL}$ streptomycin. For subsequent isolation of exosomes, cancer cells were cultured until $80 \%$ confluence and then the culture media were changed to serum-free RPMI 1640 after washing with phosphate-buffered saline (PBS) three times.

Table 1. Characteristics of the study population $(n=41)$

\begin{tabular}{ll}
\hline Variable & No. of patients \\
\hline Gender & \\
Male & 29 \\
Female & 12 \\
Age, year & \\
Median & 62 \\
Range & $46-70$ \\
Primary tumor size, cm & \\
Median & 4 \\
Range & $2.5-8$ \\
Differentiation & \\
Moderate & 35 \\
Poorly & 6 \\
Pathologic T stage & \\
T2 & 4 \\
T3 & 15 \\
T4a & 19 \\
T4b & 3 \\
Pathologic N stage & \\
N0 & 9 \\
N1a & 4 \\
N1b & 4 \\
N1c & 7 \\
N2a & \\
TNM stage & \\
IIA/B/C & \\
III B/C & \\
IVA & \\
Location & \\
Colon & \\
Rectum & \\
DFI (month) & \\
Median & \\
Range & \\
LVI & \\
Positive & \\
Negative & \\
Number of LM & \\
1 & \\
2 & \\
LM maximum size, cm & \\
\hline DFI, Disease-free interval (time between colorectal cancer surgery and pulmonary \\
metastasectomy); LM, lung metastasis. & \\
\end{tabular}

\section{Exosome isolation and characterization}

Exosomes from the culture medium were isolated using the ExoQuick-TC Exosome Precipitation Solution (System Biosciences, CA, USA) according to the manufacturer's protocol. Briefly, the culture media were centrifuged $(3000 \mathrm{~g})$ for $15 \mathrm{~min}$ to eliminate cells and large cell debris. The supernatant and ExoQuick-TC were mixed in a 5:1 ratio. Following overnight incubation at $4^{\circ} \mathrm{C}$, the exosomes were 
pelleted by centrifugation at $1500 \mathrm{~g}$ for $30 \mathrm{~min}$ and resuspended in PBS. Transmission electron microscopy (TEM) of the exosomes was performed as previously published (11). Total proteins were quantified using a Nanodrop 2000/2000c spectrophotometer (Thermo-Fisher Scientific, MA, USA).

\section{In vivo study}

BALB/c female mice (aged 6-8 weeks) were used for animal experiments. The mice were housed in a specific pathogen-free environment on a 12-h light and 12-h dark schedule with food and water. They were acclimated for at least 1 week prior to initiating in vivo studies. Ten mice were equally divided into two groups. For education experiments, five mice received $5 \mu \mathrm{g}$ of exosomes every other day, three times a week. Five micrograms of exosomes were injected into the retro-orbital venous sinus in a total volume of $50 \mu \mathrm{L}$ of PBS. Five mice received PBS as a control. The mice with and without education were sacrificed 3 weeks later. The lung tissue was analyzed. The animal experiment for this study was designed and carried out according to the standard guideline of the Institutional Animal Care and Use Committee. The study was approved by the Institutional Animal Care and Use Committee.

\section{Quantitative real-time PCR}

Total RNA was extracted from the frozen lung tissue of mouse using TRIzol (Invitrogen, USA). A PrimeScript RT Reagent Kit (TaKaRa, Japan) was used for generating cDNA. A SYBR Premix Ex Taq II Kit (TaKaRa) was used for RT-polymerase chain reaction (PCR). The primer pairs for the genes were designed using Primer Premier6. The oligonucleotide primer sequences were as follows: 5'-GAAGATCAAGATCA TTGCTCCT-3' (sense) and 5'-TACTCCTGCTTGCTG ATCCA-3' (antisense) for $\beta$-actin; 5'-ACCTCGGTG TCCTCTTGCTGTCCA-3' (sense) and 5'-GCTTGACG TTGGCTCTGGCGATGT-3' (antisense) for CXCL12. $\beta$-actin was used as an endogenous reference. The gene expression levels were calculated as $2^{-\Delta \Delta} C_{t}$ values.

\section{Statistical analysis}

The rank-sum test was used to analyze the differences in the expression of markers. The statistical analysis was performed using SPSS version 21. Statistical significance was stated as two tailed $P$ $<0.05$.

\section{Results}

\section{Expression of CXCR7, CXCR4, and CXCL12}

As shown in Figure 1, CXCR7 and CXCL12 were expressed mainly in the cytoplasm of tumor cells.
They were expressed in both primary colorectal tumors and lung metastasis. However, their expression was significantly higher in lung metastases than in primary tumors. The difference was statistically significant. As shown in Figure 1, CXCR4 was expressed mainly in the cytoplasm of tumor cells. It was expressed in both primary colorectal tumors and lung metastasis. However, the expression of CXCR4 in the two sites had no statistically significant difference $(P=0.306)$.

\section{Expression of CXCL1 2 was significantly higher in noncancerous lung tissue of CRC}

The expression of CXCL12 in the nontumor lung tissue of patients with CRC was evaluated by IHC, using benign lung disease as a control, to further investigate the role of CXCL12 in lung metastasis. The expression of CXCL12 was significantly higher in the noncancerous lung tissues of patients with CRC than in patients with benign lung disease (Fig. 2; $P<0.05$ ).

\section{Exosome identification}

Two different techniques were applied to identify the exosomes: TEM and dynamic light scattering. The diameters of exosomes derived from the culture media ranged from 30 to $100 \mathrm{~nm}$ under TEM (Fig. 3).

\section{CT26-derived exosomes increased the expression of CXCL1 2 in the lung}

BALB/c female mice (aged 6-8 weeks) were educated with exosomes isolated from CT26 to evaluate whether CT26-derived exosomes could increase the expression of CXCL12 in the lung before tumor metastasis. The mice with and without education were sacrificed 3 weeks later. The lung tissue was analyzed by the IHC method. Negative controls were run simultaneously. Interestingly, the expression of CXCL12 was found to be higher in educated mice (Fig. 4; $P<0.05$ ).

Quantitative real-time PCR was performed to confirm the results. The expression of CXCL12 was evaluated in the lung tissue from mice with and without exosome education. The expression was significantly higher in the lung tissue of mice with exosome education (Fig. 5; $P<0.01$ ).

\section{Discussion and Conclusion}

Metastasis occurs when the tumor cells leave the primary tumor and move to other organs and tissues. It means that tumor progression has reached its peak. It is also the leading cause of treatment failure [12]. Therefore, a better understanding of the mechanism of CRC metastasis may provide the new means of cancer treatment. 

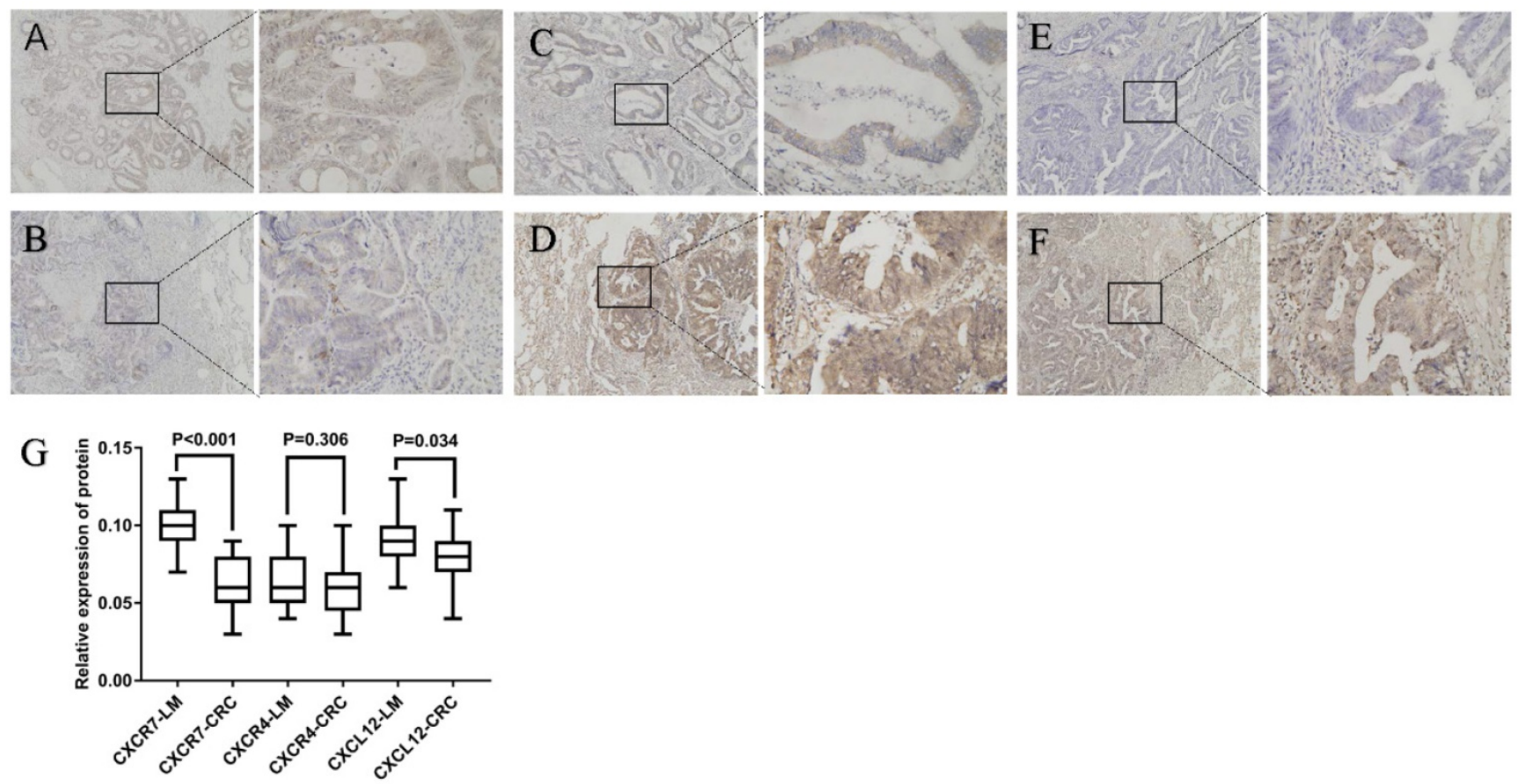

Figure 1. Expression of CXCR7, CXCL12, and CXCR4 in colorectal cancer and lung metastasis. Staining for three markers was located mainly in the cytoplasm of tumor cells. (A), (C), and (E) show the expression of CXCR4, CXCR7, and CXCL12 in primary tumors, respectively. (B), (D), and (F) show the expression of CXCR4, CXCR7, and CXCL12 in lung metastasis, respectively. The expression of CXCR7 and CXCL12 was significantly higher in lung metastases than in primary tumors. However, the expression of CXCR4 in the two sites had no statistically significant difference. (G) Relative expression of CXCR7, CXCR4, and CXCL12 protein was calculated using the density mean.
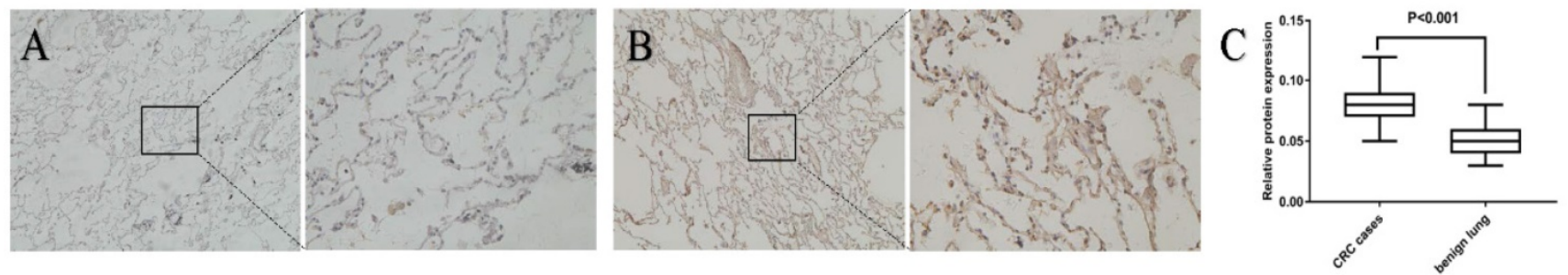

Figure 2. Expression of CXCL1 2 in noncancerous lung tissues of patients with CRC and benign lung disease. Detection of the expression of $C X C L 12$ protein assessed by immunohistochemical staining in lung tissues of patients with CRC (B) and lung tissues of patients with benign lung disease (A). CXCL12 was mainly located in the cytoplasm of alveolar epithelial cells. Noncancerous lung tissues of patients with CRC (B) had higher expression of CXCL12 compared with normal lung tissues of benign lung disease used as controls (A). (C) Relative expression of CXCL12 protein in lung tissues derived from patients with CRC and benign lung disease was calculated using the density mean.

The main function of chemokines is to direct leukocytes to the sites of injury, which is quite similar to the process of tumor metastasis. Many studies showed that chemokines could promote tumor metastasis. Muller et al. first reported that human breast cancer cells expressed a higher level of chemokine receptor CXCR4 compared with normal mammary ductal cells. The most common sites of metastasis, such as lymph node, lung, and liver, expressed its ligand CXCL12 (also known as stromal-derived factor-1 or SDF-1). A combination of CXCR4 and CXCL12 could promote tumor metastasis [13]. In this study, the expression of CXCR4, CXCR7, and CXCL12 in CRC and lung metastasis was analyzed by the IHC method. The expression of CXCR7 and CXCL12 was found to be higher in lung metastasis than in the primary site. However, the expression of CXCR4 in the two sites had no statistically significant difference. It was different from their effect in liver metastasis. Former studies reported that the expression of CXCR4 was higher in liver metastases than in primary tumors [7, 14]. Therefore, it was believed that the mechanism of liver and lung metastases was different. This was consistent with the findings of other studies. By an in vivo experiment, Guillemot et al. proved that the expression of CXCR7 on tumor might promote the growth of CRC within the lung but not within the liver [15].

This study also found that the expression of CXCL12 was significantly higher in the noncancerous lung tissue of patients with CRC than in a benign lung 
disease. It was hypothesized that the expression of CXCL12 increased before tumor metastasis. This was confirmed by further analyzing the causes of the increased expression of CXCL12.

Increasing evidence validated that exosomes could promote tumor development and metastasis (16). A previous study showed that tumor-derived exosomes could induce liver pre-metastatic niche formation and increase liver metastatic burden (17). However, whether the expression of CXCL12 in the lung could be increased by exosomes was not clear. This was confirmed through an in vivo experiment. The expression of CXCL12 was found to be higher in mice injected with tumor-induced exosomes for 3 weeks. It showed that the expression of CXCL12 was probably elevated before metastasis. This effect could be induced by exosomes. This conclusion was consistent with the concept of the pre-metastatic niche that was widely accepted to help create microenvironments suitable for colonization by metastasizing tumor cells (18). CXCL12 was another important component in the pre-metastatic niche.

In conclusion, the expression of CXCR7 and
CXCL12 was significantly higher in lung metastases than in primary tumors. Their combination could promote CRC lung metastasis. Moreover, the expression of CXCL12 was probably elevated before metastasis. This effect could be induced by exosomes.

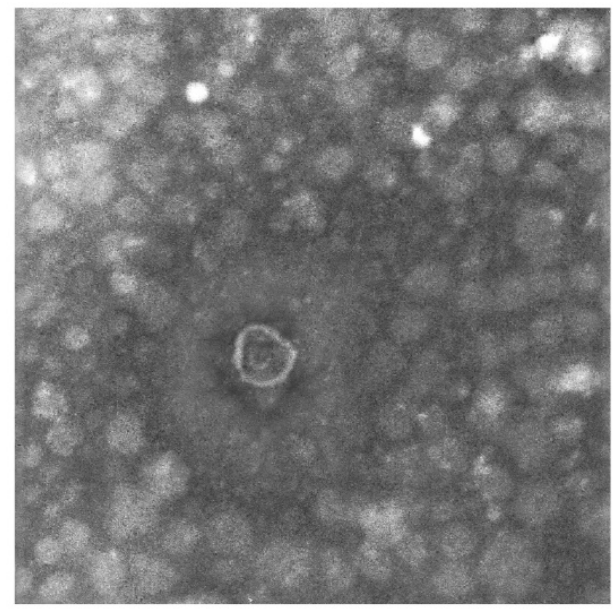

Figure 3. Identification of exosomes. Transmission electron microscopy image of CT26-derived exosomes. Arrow indicates exosomes (defined as round-shaped membrane vesicles) (size $30-100 \mathrm{~nm}$ ).
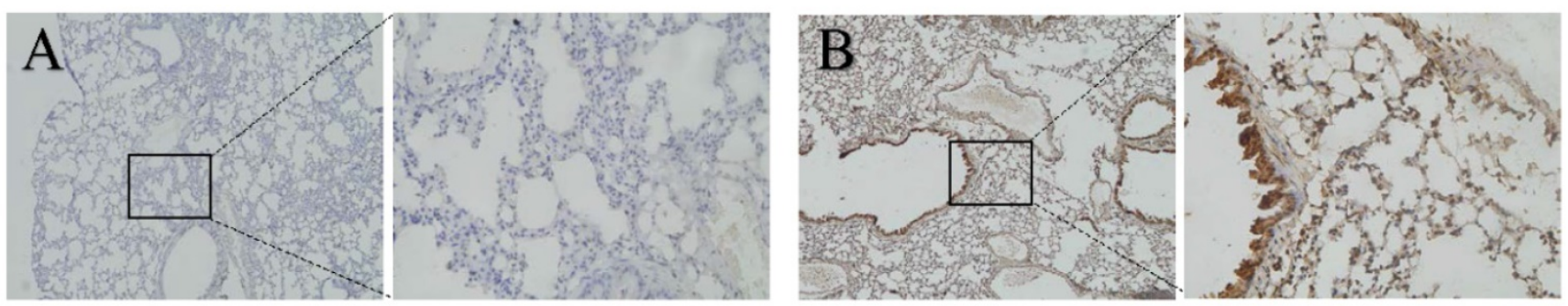

Figure 4. Expression of CXCL12 in lung tissues of mice. Detection of the expression of CXCL12 protein assessed by immunohistochemical staining in lung tissues of mice with exosome education (B) and mice without exosome education (A). Expression of CXCL12 in the lung was higher in mice with exosome education (B) than in mice without exosome education (A).

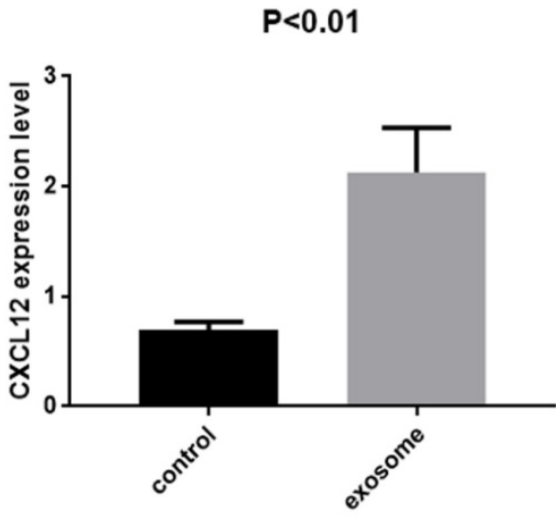

Figure 5. Expression of CXCL12 in the lung tissue of mice with and without exosome education. RT-PCR analysis of CXCL12 in CT26 cells after exosome education. The expression of CXCL12 was significantly higher in the lung tissue of mice with exosome education than in those without exosome education $(P<0.01)$.

\section{Acknowledgments}

This study was supported by the Science and Technology Support Program of the National Natural Science Foundation of China (Grant numbers: 81172373).

\section{Competing Interests}

The authors have declared that no competing interest exists.

\section{References}

1. Chen W, Zheng R, Baade PD, et al. Cancer statistics in China, 2015. CA Cancer J Clin. 2016; 66:115-32.

2. Siegel R, Naishadham D, Jemal A. Cancer statistics, 2012. CA Cancer J Clin. 2012; 62: 10-29.

3. Cho JH, Hamaji M, Allen MS, et al. The prognosis of pulmonary metastasectomy depends on the location of the primary colorectal cancer. Ann Thorac Surg. 2014; 98: 1231-1237.

4. Kim HK, Cho JH, Lee HY, et al. Pulmonary metastasectomy for colorectal cancer: how many nodules, how many times? World J Gastroenterol. 2014; 20:6133-45 
5. Rama N, Monteiro A, Bernardo JE, et al. Lung metastases from colorectal cancer: surgical resection and prognostic factors. Eur J Cardiothorac Surg. 2009; 35:444-9.

6. Kakinuma T, Hwang ST. Chemokines, chemokine receptors, and cancer metastasis. J Leukoc Biol. 2006; 79:639-51.

7. Yamada S, Shimada M, Utsunomiya $\mathrm{T}$ et al. CXC receptor 4 and stromal cell-derived factor 1 in primary tumors and liver metastases of colorectal cancer. J Surg Res. 2014; 187:107-12.

8. Miao Z, Luker KE, Summers BC, et al. CXCR7 (RDC1) promotes breast and lung tumor growth in vivo and is expressed on tumor-associated vasculature. Proc Natl Acad Sci U S A. 2007; 104:15735-40.

9. Milane L, Singh A, Mattheolabakis G, et al. Exosome mediated communication within the tumor microenvironment. J Control Release. 2015; 219: 278-94.

10. Yu DD, Wu Y, Shen HY, et al. Exosomes in development, metastasis and drug resistance of breast cancer. Cancer Sci. 2015; 106: 959-64.

11. Wei M, Yang T, Chen X, et al. Malignant ascites-derived exosomes promote proliferation and induce carcinoma-associated fibroblasts transition in peritoneal mesothelial cells. Oncotarget. 2017; 8: 42262-42271.

12. Geiger TR, Peeper DS. Metastasis mechanisms. Biochim Biophys Acta. 2009; 1796:293-308.

13. Muller A, Homey B, Hortensia Soto, et al. Involvement of chemokine receptors in breast cancer metastasis. Nature, 2001; 410:50-6.

14. Rubie C, Kollmar O, Frick VO er al. Differential CXC receptor expression in colorectal carcinomas. Scand J Immunol. 2008; 68:635-44.

15. Guillemot E, Karimdjee-Soilihi B, Pradelli E, et al. CXCR7 receptors facilitate the progression of colon carcinoma within lung not within liver. Br J Cancer. 2012; 107:1944-9.

16. Kahlert C, Kalluri R. Exosomes in tumor microenvironment influence cancer progression and metastasis. J Mol Med (Berl). 2013; 1:431-7.

17. Costa-Silva B, Aiello NM, Ocean AJ, et al. Pancreatic cancer exosomes initiate pre-metastatic niche formation in the liver. Nat Cell Biol. 2015; 17:816-26.

18. Sceneay J, Smyth MJ, Möller A.The pre-metastatic niche: finding common ground. Cancer Metastasis Rev. 2013; 32:449-64. 